\title{
Mesure de la digestion de l'azote dans l'intestin des ruminants par la technique des sachets mobiles*
}

\author{
G Piva, F Masoero, F Rossi \\ Università cattolica sacro cuore, facolta di agraria, Istituto di scienze della nutrizione, \\ Via Emilia Parmense 84, 29100 Piacenza, Italie
}

\begin{abstract}
Summary - Measurements of the nitrogen digestion in the intestine of ruminants by the mobile bag technique. Forty-two feeds frequently used in livestock feeding were tested to provide indications regarding the digestive use of nitrogen in ruminants. Three milking cows provided with ruminal and intestinal fistula were used; and the mobile bag technique adopted, originally proposed by INRA.
\end{abstract}

L'évaluation de la valeur azotée des aliments en protéines digestibles dans l'intestin (PDI) (INRA, 1988) suppose la connaissance de la dégradabilité théorique dans le rumen $(D T)$ des protéines alimentaires et de leur digestibilité réelle $(d r)$ dans l'intestin. Si la bibliographie concernant la $D T$ est abondante, les données concernant la $d r$ sont peu nombreuses et les méthodes de prévision ne sont pas encore standardisées. Cette étude concerne la mesure de la $d r$ de 42 aliments d'intérêt zootechnique, par la technique des sachets mobiles proposée par Peyraud et al (1988).

Matériel et méthodes - Les aliments étudiés figurent dans le tableau I. Les mesures ont été réalisées avec 3 vaches primipares du type Pie Noire, au $5^{\mathbf{e}}$ mois de lactation, produisant $23 \mathrm{~kg}$ de lait par jouren moyenne. Elles étaient munies d'une canule du rumen et d'une canule du duodénum. Elles recevaient 2 fois par jour (à $8 \mathrm{~h}$ et $17 \mathrm{~h}$ ) un régime à $14,5 \%$ de matières azotées totales composé, sur la base de la matière sèche, de $22 \%$ d'ensilage de maìs, $44 \%$ de foin et $34 \%$ d'aliment concentré. Le niveau des apports énergétiques était de 2,5 fois l'entretien. Les aliments, broyés à la grille de $1 \mathrm{~mm}$, ont été introduits dans des sachets de nylon $(10 \times 15 \mathrm{~cm})$ en quantité relativement élevée $(5 \mathrm{~g})$ de façon à ce que la quantité de résidu suffise à l'étape ultérieure de la mesure. Après $15 \mathrm{~h}$ de séjour dans le rumen, les sachets ont été lavés et lyophilisés. Leur contenu a ensuite été introduit dans des petits sachets de nylon $(6 \times 3 \mathrm{~cm}$, pores de $46 \mu \mathrm{m})$ qui ont été plongés pendant $2,5 \mathrm{~h}$ dans une solution de pepsine et d'acide chlorhydrique, puis introduits à $17 \mathrm{~h}$ dans l'intestin. Les sachets ont été recueillis dans les fèces en moyenne $19 \mathrm{~h}$ après leur introduction dans l'intestin. Les mesures ont été répétées $9-20$ fois par aliment.

\footnotetext{
* Recherche réalisée avec contribution MPI $40 \%$.
} 
Tableau I. Digestibilité réelle $(d r)$ dans l'intestin des matières azotées totales (MAT) de différents aliments, non disparues après un séjour de $15 \mathrm{~h}$ dans le rumen.

\begin{tabular}{lcc}
\hline Aliments & $\begin{array}{c}\text { MAT } \\
(\%) M S\end{array}$ & $\begin{array}{c}d r \\
(\%)\end{array}$ \\
\hline Maïs & 9,6 & 88,7 \\
T de germe de maïs & 19,2 & 94,1 \\
Gluten de maïs & 66,1 & 83,1 \\
G de soja 1 & 35,7 & 98,8 \\
G de soja 2 & 42,0 & 99,3 \\
G de soja extrudée & 41,6 & 87,5 \\
G de soja extrudée 2 & 39,5 & 97,9 \\
G de soja en flocons 1 & 41,3 & 82,3 \\
G de soja en flocons 2 & 38,8 & 95,8 \\
T de soja pression & 40,8 & 98,0 \\
T de soja déshuilé & 49,9 & 98,8 \\
Farine de viande 1 & 53,5 & 81,5 \\
Farine de viande 2 & 56,3 & 84,8 \\
Farine de poisson 1 & 71,6 & 94,6 \\
Farine de poisson 2 & 79,0 & 97,4 \\
Farine de plumes & 82,3 & 96,2 \\
Farine de sang & 92,6 & 63,2 \\
Pois & 25,5 & 99,5 \\
Pois chiche & 29,4 & 98,3 \\
Féverole & 18,1 & 99,6 \\
Orge & 12,2 & 97,6 \\
Luzerne déshydr & 19,8 & 80,6 \\
Foin de luzerne & 19,4 & 90,4 \\
Ensilage d'herbe & 16,5 & 89,8 \\
Ensilage luzerne préf & 16,5 & 90,6 \\
T de tournesol & 36,7 & 98,6 \\
T de coton & 34,8 & 94,6 \\
G de coton & 20,4 & 95,8 \\
G de coton extrudées & 22,2 & 93,2 \\
Son de blé 1 & 18,4 & 98,2 \\
Son de blé 2 & 17,1 & 93,1 \\
Farine basse 1 & 18,7 & 99,2 \\
Farine basse 2 & 19,8 & 98,2 \\
Son fin 1 & 15,8 & 96,4 \\
Son fin 2 & 15,7 & 96,2 \\
Remoulage bis 1 & 16,9 & 97,9 \\
Remoulage bis 2 & 19,6 & 96,9 \\
T d'arach traité & 51,7 & 99,0 \\
Drêches de grain & 24,6 & 97,2 \\
T de coprah & 22,2 & 90,0 \\
T de lin & 34,6 & 92,1 \\
Drêches de brass & 19,1 & 88,2 \\
\hline T & & \\
\hline & &
\end{tabular}

$T=$ tou teau; $G=$ graine .
Résultats et discussion - Les mesures de $d r$ ont des écarts types faibles et sont donc relativement répétables. La comparaison de nos données de $d r$ (tableau I) avec celles obtenues par Peyraud et al (1988) montre une remarquable similitude, bien que les régimes des animaux dans les 2 expériences soient très différents. Seuls le maïs et le gluten de maïs font exception : dans notre essai, leur $d r$ est respectivement de $88,7 \%$ et $83,1 \%$, contre $98,4 \%$ et $99,9 \%$ pour Peyraud et al (1988), et $86,2 \%$ et $100 \%$ pour Voigt et al (1985), dont les vaches étaient alimentées au niveau de l'entretien. Cette différence est probablement liée au fait que la dégradation de I'N de ces aliments dans le rumen est faible et peut, par conséquent, être influencée par la richesse du régime alimentaire en énergie. Les farines de viande et de sang présentent les valeurs de $d r$ les plus faibles, tandis que celles du soja varient de façon importante avec les traitements technologiques. La similitude de nos données de $d r$ avec celles de Peyraud et al (1988) permet d'adopter leur résultats, obtenus sur des animaux à l'entretien, pour des animaux en production.

En conclusion, la technique des sachets mobiles dans l'intestin peut être répétée dans des conditions variées en donnant toujours des résultats très similaires.

Peyraud JL, Genest-Rulquin C, Vérité R (1988) Reprod Nutr Dév 28 (suppl 1), 129-130

Voigt J, Piatkowski B, Engelman $\mathrm{H}$, Rudolph E (1985) Arch Tierernehr 35, 555-562 